\title{
A plataforma Moodle no treinamento de estudantes de Odontologia na avaliação de restaurações classe II em resina composta
}

\author{
Eliane Cristina Gava Pizi*; Ana Beatriz Millan Sasso**; Millena Queiroz Cavalheiro**; Thiago Henrique \\ Scarabello Stape*; Claudia de Oliveira Lima Coelho***; Graziela Ávila Prado Galhano**** \\ * Doutor, professor do Departamento de Clínica Odontológica \\ da Faculdade de Odontologia da UNOESTE \\ ** Graduada pela Faculdade de Odontologia da UNOESTE \\ *** Mestre, professor do Departamento de Clínica Odontológica \\ da Faculdade de Odontologia da UNOESTE \\ **** Post-Doc, professor do Departamento de Clínica \\ Odontológica da Faculdade de Odontologia da UNOESTE
}

Recebido em 01/11/2016. Aprovado em 23/01/2017.

\begin{abstract}
RESUMO
O objetivo do estudo foi analisar o padrão de conduta de acadêmicos de Odontologia frente a restaurações classe II em resina composta cuja situação clínica seja de caráter duvidoso. Deste modo, pode-se detectar dificuldades comuns dos estudantes na tomada de decisão da conduta clínica, por meio de critérios de avaliação padronizados, bem como aperfeiçoar uma metodologia auxiliar de ensino digital baseada na plataforma Moodle. Participaram da pesquisa 65 estudantes da disciplina de Dentística que já haviam recebido treinamento teórico sobre critérios clínicos para avaliação de restaurações conforme FDI (World Dental Federation). A coleta de dados foi feita por meio de questionários disponíveis no site Aprender Unoeste (plataforma Moodle). Os estudantes analisaram 22 imagens de restaurações classe II em resina composta disponíveis no site observando os seguintes itens: parâmetros estéticos (brilho superficial, manchamento superficial e marginal, correspondência de cor e translucidez), parâmetros funcionais (fratura do material e retenção, adaptação marginal, forma anatômica proximal/contorno) e parâmetros biológicos (recorrência de cáries e integridade dental). A média de acertos dos alunos foi $6,3 \pm 0,7$ (sobre pontuação máxima $=10$ ), sendo que os parâmetros estéticos apresentaram menor percentual de acertos. Após o preenchimento do questionário os estudantes obtinham o feedback imediato de suas avaliações, tendo então a possibilidade de verificar seus erros e qual seria a resposta adequada. $\mathrm{O}$ estudo detectou dificuldades dos estudantes de Odontologia na análise de restaurações em resina composta e possibilitou que estes adquirissem experiência visual clínica, desenvolvendo seu senso crítico, como auxílio de novas metodologias de ensino-aprendizagem.
\end{abstract}

Descritores: Resinas Compostas. Restauração Dentária Permanente. Falha de Restauração Dentária. Educação Superior. 


\section{INTRODUÇÃO}

$\mathrm{Na}$ clínica diária, é frequente que estudantes de Odontologia e cirurgiões dentistas se deparem com restaurações posteriores em resina composta que deixam muitas dúvidas quanto à necessidade de reparo ou de substituição. Manchamentos do corpo da restauração e da margem cavosuperficial, rugosidade superficial, presença de fendas marginais, cárie secundária, forma anatômica desviada do normal, impacção alimentar, sensibilidade pós-operatória e fratura da restauração ou do tecido dental são ocorrências a curto, médio e longo prazo mais frequentemente observadas em compósitos utilizados em dentes posteriores $^{1,2}$.

A indicação para a substituição de restaurações está diretamente relacionada a critérios subjetivos e, muitas vezes, de difícil definição entre estudantes, avaliadores e professores; sendo o manchamento do corpo e da margem cavosuperficial da restauração o principal motivo de substituição de restaurações de resina composta ${ }^{3}$.

Tradicionalmente, a substituição era a abordagem ideal para o tratamento de restaurações de resina composta com defeito, no entanto, o reparo de compósitos oferece uma alternativa mais conservadora, pois as restaurações são, em parte ainda mantidas ${ }^{4}$. O reparo da restauração tem a vantagem de levar menos tempo clínico e pode por vezes ser realizado sem a utilização de anestesia local, sendo, por consequência, menos desconfortável para o paciente quando comparado com a substituição ${ }^{5,6}$. Profissionais que não consideram o reparo antes de tomar a decisão de substituir restaurações que apresentam pequenos defeitos devem ser encorajados a considerar a inclusão de reparo nas opções de tratamento. Ao analisar e considerar estas medidas, em primeiro lugar, deve-se considerar monitoramento, renovação e reparo. A substituição da restauração deve ser o último recurso, quando não há alternativa viável $^{6}$.

A resistência de união de reparos em resinas compostas tem apresentado resultados satisfatórios para que o reparo seja uma opção de tratamento na clínica diária. Deve-se realizar o tratamento mecânico da superfície da restauração, a fim de remover a camada superficial deteriorada, criar micro retenções e aumentar a energia de superfície, para posterior aplicação do silano e do adesivo ${ }^{7}$. Embora o reparo de restaurações seja ensinado e praticado há vários anos, poucos estudos clínicos controlados sobre a longevidade das restaurações reparadas são publicados.

Em 1980 Ryge publicou critérios clínicos para avaliação de restaurações, tendo ampla aceitação da comunidade científica mundial. Esses critérios ficaram conhecidos como critérios da USPHS (United States Public Health Service) sendo empregado e adaptado por diversos autores com o decorrer dos anos até recentemente. Entretanto, conforme o avanço no desempenho clínico dos materiais restauradores e pela sensibilidade limitada dos critérios Ryge para detectar essas novas mudanças, se percebeu a necessidade criar uma abordagem mais detalhada e sensível ${ }^{2}$.

Em 2007, Hickel et al. ${ }^{1}$ publicaram novos critérios clínicos para a avalição de restaurações, sendo aprovado pela FDI (World Dental Federation), ficando conhecidos como critérios clínicos da FDI, que em 2010 foram atualizados por Hickel et $\mathrm{al.}^{2}$. Os critérios foram categorizados em três grupos: (1) parâmetros estéticos, (2) parâmetros funcionais, e (3) parâmetros biológicos. Cada critério pode ser expresso em cinco pontuações: três para aceitável e dois para não aceitável.

Em 2008 foi disponibilizada a ferramenta online e-calib (www.e-calib.info ) para 
treinamento e calibração de pesquisadores e também para auxiliar no aprendizado de acadêmicos de Odontologia. A ferramenta é baseada na plataforma Moodle (www.moodle. $\underline{\text { com) }}$ e pode ser utilizada de forma gratuita, desde que o usuário se registre. Os casos são selecionados aleatoriamente a partir de um vasto banco de dados apenas com fotografias de alta qualidade dos casos clínicos. As respostas dadas pelo participante são verificadas pelo sistema quanto à concordância com a classificação dada por uma equipe de quatro peritos ${ }^{2}$.

Nas universidades, em qualquer tipo de faculdade ou instituto de pesquisa, é possível observar as inúmeras aplicações da informatização: gerenciamento de banco de dados, organização de catálogos, agilização de diagnósticos, entre outras. Dentro desse contexto, o ensino a distância pode facilitar esse aprendizado, porém os alunos, futuros profissionais, precisam estar preparados para lidar com as novas ferramentas disponibilizadas, usando-as em seu benefício e, consequentemente, em sua capacitação profissional, para a garantia de maior produtividade e interação com as diversas esferas no campo de trabalho ${ }^{8}$.

O uso da plataforma Moodle no treinamento de estudantes tem sido altamente difundido entre educadores do mundo inteiro. $\mathrm{O}$ Moodle é um Sistema open source de Gerenciamento de Cursos, também conhecido como Learning Management System ou um Ambiente Virtual de Aprendizagem (AVA). A palavra Moodle é originalmente um acrônimo para Modular Object-Oriented Dynamic Learning Environment (Ambiente de Aprendizagem Dinâmico Modular Orientado a Objeto), útil principalmente para programadores $\mathrm{e}$ profissionais da educação? .

Nas avaliações clínicas de restaurações de resina composta, vários fatores estão associados com o aumento de falha de restaurações. A falha aumenta à medida que aumenta o número de superfícies na restauração ${ }^{3}$. Restaurações mais extensas e com maior número de faces, apresentam também maior porcentagem de falhas. Restaurações classe II têm de 50 a $70 \%$ mais falhas que classe I, possivelmente pelo tamanho da restauração e pela maior dificuldade da técnica restauradora ${ }^{10}$.

Como o desgaste das restaurações em resina podem ser acompanhados por erupção passiva, ou pelo menos inclinação do dente antagonista, um exame clínico cuidadoso deve ser sempre realizado nas avaliações clínicas ${ }^{6}$, a fim de impedir que danos biológicos a médio e longo prazo possam ocorrer, inclusive levando à perda do elemento dental.

Sabendo-se que restaurações diretas em resina composta em dentes posteriores, especialmente envolvendo a face proximal, apresentam alta complexidade de execução e inúmeras possibilidades de falhas que vão desde a escolha inadequada do material restaurador, técnica adesiva, cuidados com a fotoativação e a inserção da resina propriamente dita, o cirurgiãodentista deve estar atento aos possíveis problemas decorrentes durante um exame clínico criterioso. Este estudo justifica-se na dificuldade que acadêmicos de Odontologia enfrentam na tomada da decisão terapêutica frente a restaurações de resina composta que se deparam na prática clínica diária. Deste modo, a complementação do ensino de Dentística com recursos auxiliares digitais aliada a critérios clínicos padronizados pode vir a suprir essa demanda por padronização de avaliação clínica de restaurações e por experiência visual clínica.

Assim, o objetivo deste estudo foi analisar o padrão de conduta de acadêmicos de Odontologia frente a restaurações classe II em resina composta cuja situação clínica seja de caráter duvidoso. Deste modo, pode-se detectar dificuldades comuns dos estudantes na tomada 
de decisão da conduta clínica através de critérios de avaliação padronizados, bem como aperfeiçoar uma metodologia auxiliar de ensino digital baseada na plataforma Moodle.

\section{MATERIAL E MÉTODOS}

A pesquisa teve início após a aprovação pelo Comitê de Ética em Pesquisa da Universidade do Oeste Paulista (UNOESTE), tendo como público alvo todos os acadêmicos de Odontologia do $6^{0}$ termo, regularmente matriculados na disciplina de Dentística Clínica II. Entre os critérios de exclusão estava a não assinatura voluntária do termo de consentimento livre e esclarecido, assim como a não participação do aluno no treinamento teórico prévio à avaliação.

Foram apresentados e discutidos com os 65 participantes, os critérios clínicos de avaliação de restaurações conforme a FDI (Federação Dentária Internacional), elaborados por Hickel et al. $(2007)^{1}$ e atualizados em 2010². Este momento contou com uma exposição teórica dos conceitos e exemplificação com as fotos disponíveis no site www.e-calib.info, dos mesmos autores.

Em um segundo momento, os estudantes foram orientados a realizar uma avaliação de imagens de restaurações classe II em resina composta disponíveis na plataforma Moodle (www.moodle.com), através do site Aprender Unoeste (www.unoeste.br/site/ava/).

As imagens das restaurações, de fonte própria da orientadora da pesquisa, foram selecionadas aleatoriamente e reunidas durante a prática da disciplina de Dentística Clínica. As fotografias foram obtidas com câmera fotográfica digital Canon (Canon Inc, Tokyo, Japão), com objetiva macro 100 contando com o auxílio de espelho intraoral para fotografia oclusal e bucal de adulto e também de afastadores bucais (figura 1). Para se chegar à resposta correta das imagens, dois examinadores experientes realizaram antes uma calibração e treinamento no próprio site www.e-calib.info e foram selecionadas as 22 imagens que obtiveram $100 \%$ de concordância entre os avaliadores.

Para a análise das imagens, observaram os seguintes parâmetros: estéticos (brilho superficial, manchamento superficial e marginal, correspondência de cor e translucidez), funcionais (fratura do material e retenção, adaptação marginal, forma anatômica proximal/contorno) e biológicos (recorrência de cárie e integridade dental). Cada critério pode ser expresso com cinco pontuações, sendo: três para aceitável, (1) clinicamente excelente/muito bom, (2) provavelmente muito bom após polimento e (3) clinicamente suficiente/ satisfatório (com defeitos aceitáveis, porém não reparáveis); e dois para não aceitável, (4) reparável e (5) substituição necessária (quadro 1).

Os estudantes recebiam o feedback imediato das avaliações e os resultados eram computados conforme o número de acertos em cada avaliação realizada.

\section{RESULTADOS}

Observou-se o padrão de conduta de acadêmicos de Odontologia frente a restaurações em resina composta classe II escolhidas aleatoriamente, bem como complementou-se o ensino da disciplina de Dentística com recursos auxiliares aliados a critérios clínicos padronizados.

Ao se realizar a avaliação das imagens na plataforma Moodle, atribuiu-se uma nota para cada resposta correta. Também foram parcialmente consideradas respostas que coincidissem com o padrão de aceitabilidade do gabarito. Por exemplo, se o estudante respondesse que a restauração apresentava escore (1) clinicamente excelente, mas o gabarito correto era (3) clinicamente suficiente, por 
enquadrarem-se dentro do padrão "aceitável", a resposta era considerada, mas com uma pontuação menor na nota final do estudante.

As respostas alcançaram média de acertos de 6,3 $\pm 0,7$ (tabela 1), correspondendo a $63 \%$ de acertos no geral. Os parâmetros estéticos
$(56,8 \%)$ apresentaram menor porcentagem de acertos que os biológicos $(66,9 \%)$ e funcionais $(66,3 \%)$, sendo os critérios manchamento superficial, brilho superficial e adaptação marginal os que apresentaram menor percentual de acertos na média.

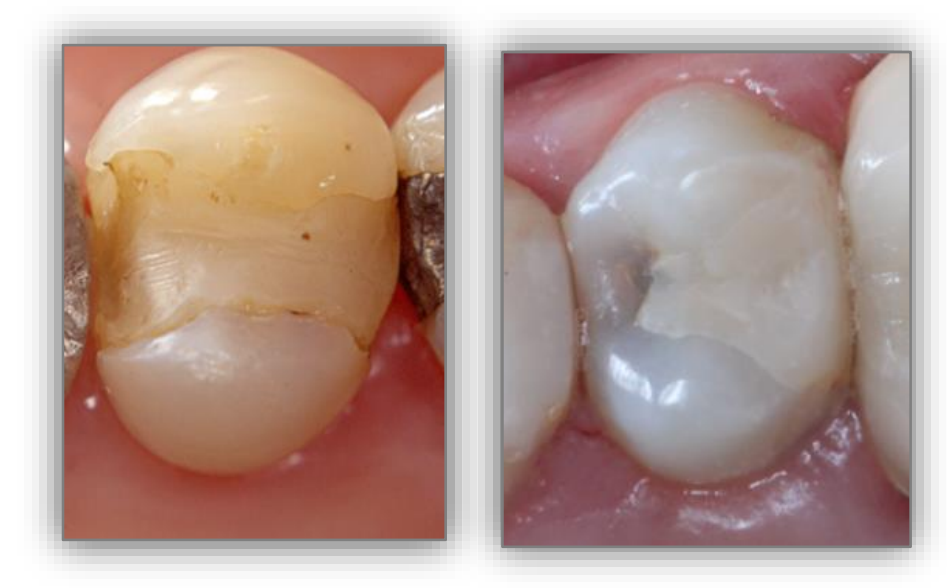

Figura 1. Exemplos das imagens disponíveis para avaliação.

Quadro 1. Parâmetros avaliados nas fotografias (modificado de Hickel et al. ${ }^{1,2}$ ).

\begin{tabular}{|c|c|c|c|}
\hline \multicolumn{2}{|r|}{ Parâmetros avaliados } & \multicolumn{2}{|r|}{ Pontuações } \\
\hline 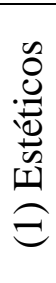 & $\begin{array}{ll}\checkmark & \text { brilho superficial } \\
\checkmark & \text { manchamento superficial e } \\
\text { marginal } \\
\checkmark \\
\text { correspondência de cor e } \\
\text { translucidez }\end{array}$ & \multirow{2}{*}{ 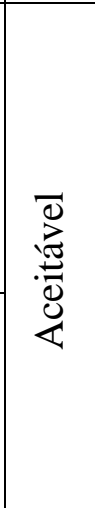 } & $\begin{array}{l}\text { (1) clinicamente } \\
\text { excelente/muito bom } \\
\text { (2) provavelmente muito bom } \\
\text { após polimento } \\
\text { (3) clinicamente suficiente/ } \\
\text { satisfatório (com defeitos }\end{array}$ \\
\hline \multirow{2}{*}{ 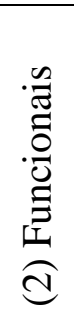 } & \multirow[t]{2}{*}{$\begin{array}{ll}\checkmark & \text { fratura do material e retenção } \\
\checkmark & \text { adaptação marginal } \\
\checkmark & \text { forma anatômica } \\
& \text { proximal/contorno }\end{array}$} & & $\begin{array}{l}\text { aceitáveis, porém não } \\
\text { reparáveis) }\end{array}$ \\
\hline & & & $\begin{array}{l}\text { (4) reparável } \\
\text { (5) substituição necessária }\end{array}$ \\
\hline 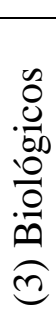 & $\begin{array}{ll}\checkmark & \text { recorrência de cáries } \\
\checkmark & \text { integridade dental }\end{array}$ & 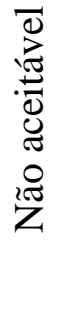 & \\
\hline
\end{tabular}


Tabela 1. Percentuais de acertos por dente restaurado, organizados por critério e parâmetros analisados.

\begin{tabular}{cccc}
\hline Dente & \% de acertos & Critério analisado & Parâmetro \\
\hline 1 & 52,9 & Brilho superficial & Estético \\
\hline 11 & 36,8 & Brilho superficial & Estético \\
\hline 20 & 75,0 & Brilho superficial & Estético \\
\hline 2 & 39,7 & Manchamento superficial & Estético \\
\hline 12 & 60,3 & Manchamento superficial & Estético \\
\hline 16 & 79,4 & Manchamento marginal & Estético \\
\hline 6 & 57,4 & Correspondência de cor e translucidez & Estético \\
\hline 21 & 52,9 & Correspondência de cor e translucidez & Estético \\
\hline 3 & 73,5 & Fratura do material e retenção & Funcional \\
\hline 7 & 73,5 & Fratura do material e retenção & Funcional \\
\hline 4 & 61,8 & Adaptação marginal & Funcional \\
\hline 8 & 61,8 & Adaptação marginal & Funcional \\
\hline 13 & 63,2 & Adaptação marginal & Funcional \\
\hline 22 & 30,9 & Adaptação marginal & Funcional \\
\hline 5 & 69,1 & Funcional \\
\hline 9 & 77,9 & Forma anatômica proximal/contorno & Funcional \\
\hline 14 & 88,2 & Forma anatômica proximal/contorno & Funcional \\
\hline 17 & 63,2 & Forma anatômica proximal/contorno & Funcional \\
\hline 10 & 52,9 & Recorrência de cáries & Biológico \\
\hline 15 & 30,9 & Recorrência de cáries & Biológico \\
\hline 18 & 95,6 & Recorrência de cáries & Biológico \\
\hline 19 & 88,2 & Integridade dental & Biológico \\
\hline
\end{tabular}

Na figura 2, exemplo de feedback dado aos alunos ao responderem as questões, ilustrando as duas possíveis respostas para o critério solicitado (contorno proximal). Apesar de ser classificada como escore 5 (inaceitável e necessitando substituição), poderia ainda sofrer um reparo na face proximal (mas ainda classificada como inaceitável dentro deste critério).

\section{DISCUSSÃO}

Sabe-se que existe grande divergência na avaliação de restaurações para tomada de decisão clínica, mesmo sendo este um passo relevante no sucesso do tratamento. Esta avaliação pode ser ainda influenciada por diversos fatores, mas deve-se tentar diminuir a subjetividade deste processo com a aplicação de critérios padronizados.
O uso de Tecnologias da Informação e Comunicação (TICs) coopera para o desenvolvimento tanto na forma presencial como à distância, explorando o uso da imagem, som, movimento simultâneo, a máxima velocidade no atendimento às demandas e o trabalho com as informações em tempo real ${ }^{11}$. O uso do vídeo e outros recursos eletrônicos (retroprojetores, slides e informática) auxilia nos resultados pedagógicos positivos decorrentes do processo de ensino e aprendizagem. Em especial na Odontologia, os alunos apresentam dificuldade de visualização e localização espacial das estruturas e tecidos, desse modo, a utilização de outras técnicas de ensino, bem como sua disponibilização na internet, incentiva o relacionamento teórico/prático do aluno, facilitando o aprendizado $^{12}$. Por essa facilidade, a 
implementação da informática é de extrema importância na formação de novos profissionais ${ }^{13}$. Percebe-se com a utilização destes recursos, que passa-se a trabalhar em um ambiente que é muito familiar aos jovens, transpondo muitas vezes a barreira existente entre professor e aluno.

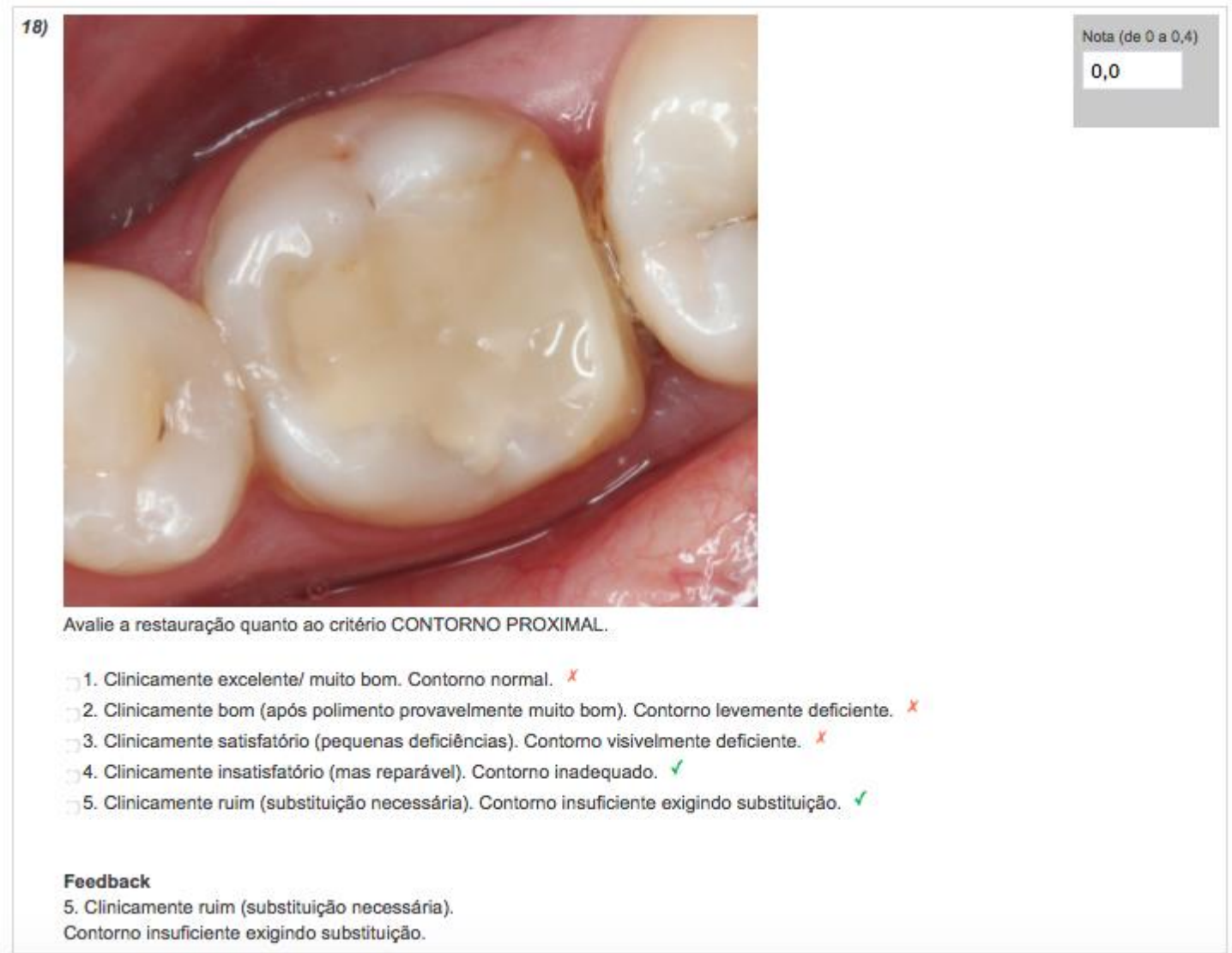

Figura 2. Exemplo de feedback para questão respondida.

Santos et al. ${ }^{14}$ (2008) avaliaram 76 estudantes de odontologia matriculados na disciplina de Dentística quanto à necessidade de manutenção, reparação ou substituição de restaurações de amálgama e resina composta por meio de fotografias. Os autores chegaram à conclusão de que há uma grande variabilidade no padrão de conduta quanto à decisão de trocar, reparar ou substituir restaurações entre os estudantes avaliados. Esta variabilidade pode ser minimizada com treinamentos baseados em critérios padronizados e definidos internacionalmente, por isto neste estudo seguiuse os apresentados por Hickel et al. em 2007¹.

Quanto à quantidade de acertos encontrados neste estudo, fica claro que estudantes de Odontologia, pela própria falta de experiência, tenham dificuldade em realizar o exame clínico e detectar falhas em restaurações em resinas compostas. O treinamento virtual serve justamente para possibilitar um treinamento extra e propiciar maior segurança ao iniciar o atendimento clínico. Os exercícios realizados logo após a etapa de treinamento teórico, podem ainda ser disponibilizados aos estudantes, a qualquer momento que o docente ache necessário reforçar o treinamento de avaliação clínica.

Outro ponto importante a ser discutido é o feedback, momento que o estudante tem a chance 
de confirmar se sua resposta está correta. O método auxilia no processo ensinoaprendizagem a medida que propicia um retorno imediato de seu erro na avaliação.

Para a implementação de novas metodologias, os desafios ainda são muitos, temse que assegurar a difusão de novas tecnologias para melhorar os modos pelos quais o conhecimento pode ser produzido, administrado, difundido e acessado. A adoção de novas abordagens, novas formas de ensinar e de fazer aprender requerem estudo, dedicação, compromisso e ousadia para inovar. Entretanto, quando se tratam de novas práticas didáticas, pode-se dizer que já vem sendo alcançado o mais importante do objetivo docente: o de sentir os alunos mais motivados, mais receptivos e pró$\operatorname{ativos}^{15}$.

Tem-se que tomar o cuidado no preparo dos docentes, para que a plataforma Moodle não sirva apenas como um repositório de material didático e haja pouca interação pedagógica e construção de conhecimento dentro deste ambiente $^{16}$. Estes treinamentos na plataforma Moodle vem justamente no sentido do favorecimento do ensino-aprendizagem onde o estudante seja parte ativa deste processo.

Em Odontologia a introdução de TICs e AVA podem potencializar a interatividade no processo ensino-aprendizagem. $\mathrm{O}$ contato com alunos através do ambiente virtual, em atividades semipresenciais, também tem se mostrado uma alternativa que auxilia nas aulas em laboratório e na sedimentação de conhecimentos ${ }^{17}$. Apesar deste estudo abordar as avaliações de restaurações em resina composta, ele pode ser utilizado em diversas outras situações em Odontologia. O mesmo tipo de treinamento e exercícios já vem acontecendo na disciplina de Clínica para treinamento de detecção de lesões cariosas em superfície oclusal.

Nos dias de hoje, pretende-se formar um profissional que deve ser capaz de desenvolver habilidades humanas e profissionais, ou seja, aprender o que pode ser feito com conhecimentos adquiridos: aplicá-los, resolver problemas, criar situações, usá-los em situações novas e diferentes ${ }^{11}$. Acredita-se que avaliações e treinamentos como o realizado neste estudo, possam alcançar estes objetivos pois possibilitam o desenvolvimento de habilidades profissionais onde o estudante é capaz de aplicar os conhecimentos adquiridos para resolver problemas, desenvolvendo seu senso crítico. Desse modo, o aluno de Odontologia se sente mais seguro tanto na prática clínica acadêmica quanto na profissional.

\section{CONCLUSÃO}

O estudo detectou dificuldades dos estudantes de Odontologia na análise de restaurações em resina composta e possibilitou que estes adquirissem experiência visual clínica, desenvolvendo seu senso crítico, por meio de novas metodologias de ensino-aprendizagem.

\section{ABSTRACT \\ Dental students training on the evaluation of class II composite restorations using the Moodle platform}

The study aimed to evaluate the clinical conduct of dental undergraduate students regarding unsatisfactory Class II composite restorations with doubtful prognosis, in order to detect their difficulties and to improve learning methods using a Moodle online platform. A total of 65 sixth semester students were enrolled, they were attending Operative-Restorative Dentistry discipline and received previous theoretical training on restorations evaluation according FDI (World Dental Federation) criteria and randomly selected as volunteers. Data was collected using a questionnaire available on a specific online Moodle platform. Students analyzed 22 digital images of class II composite restorations observing aesthetic parameters (i.e., surface gloss, surface and marginal staining, color matching and translucency), anatomical aesthetic 
form, functional parameters (i.e., restoration fracture and retention, marginal adaptation, proximal anatomical shape/contour) and biological parameters (i.e., recurrence of caries and tooth integrity). The maximum individual score for correct answers was established as 10 points. Immediate feedback was provided allowing students to check the appropriate responses once the questionnaire was finished. The average of correct answers was $6.3(0,7$ standard deviation). Graduation students present a considerable high difficulty to determine the clinical success of composite restorations. The proposed new teaching/learning methodology enhances the visual experience and the critical contributes to accelerate the learning curve on this topic.

Descriptors: Composite Resins. Dental Restoration, Permanent. Dental Restoration Failure. Education, Higher.

\section{REFERÊNCIAS}

1. Hickel R, Roulet JF, Bayne S, Heintze SD, Mjör IA, Peters M, et al. Recommendations for conducting controlled clinical studies of dental restorative materials. Clin Oral Investig. 2007;11(1):5-33.

2. Hickel R, Peschke A, Tyas M, Mjör I, Bayne S, Peters M, et al. FDI World Dental Federation: Clinical criteria for the evaluation of direct and indirect restorations-update and clinical examples. Clin Oral Investig. 2010;14(4):349-66.

3. Dubinski P, Cardoso SDA, Hoeppner MG. Avaliação das causas das substituições de restaurações nas nas disciplinas de Dentística II e Clínica Integrada do curso de Odontologia da Unipar - campus Umuarama. UEPG Ci Biol Saúde. 2005;11(1):7-14.

4. Silva EF, Batista A, Mello D, Lisboa J, Fonseca R, Mathias P. Reparo de restauração de resina composta : revisão de literatura e apresentação de caso clínico. Rev Bahiana Odontol. 2013;4(1):65-75.

5. Catleugh M, Merry A, Tickle M, Dunne SM, Brunton P. Replacement versus repair of defective restorations in adults : resin composite. Cochrane Database Syst Rev. 2010;17(2):1-7.

6. Blum IR, Lynch CD, Wilson NH. Factors influencing repair of dental restorations with resin composite. Clin Cosmet Investig Dent. 2014;6:81-7.

7. Bacchi A, Cavalcante LMA, Scheneider LFJ, Consani RLX. Reparos em restaurações de resina composta - revisão de literatura. RFO. 2010;15(3):331-5.

8. Dotta EAV, Garcia PPNS, Candido LM. Elaboração de um curso interativo voltado ao aprendizado de um sistema aplicativo em Odontologia, utilizando a Plataforma Moodle. Rev Odontol Univ Cid São Paulo. 2012;24(1):6-14.

9. Sabbatini R. Ambiente de Ensino e Aprendizagem via internet: a plataforma moodle. São Paulo Inst Edu Med. 2007. (Acesso em: 15 jan 2017). Disponível em: http://www.ead.edumed.org.br/file.php/ 1/PlataformaMoodle.pdf

10. McCracken MS, Gordan VV, Litaker MS, Funkhouser E, Fellows JL, Shamp DG, et al. A 24-month evaluation of amalgam and resin-based composite restorations: Findings from the National Dental PracticeBased Research Network. J Am Dent Assoc. 2013 Jun;144(6):583-93.

11. Masetto MT. Inovação na aula universitária: espaço de pesquisa, construção de conhecimento interdisciplinar, espaço de aprendizagem e tecnologias de comunicação. Perspectiva. 2011;29(2):597-620.

12. Borges AB, Pucci CR, Torres CR, Barcellos DC. Utilização de vídeo como recurso complementar de ensino em dentística operatória. Brazilian Dent Sci. 2009;12(3):6-10.

13. Machado RPA, Scherma AP, Pisa IT. Uso da informática na Odontologia. Clipe Odonto. 2012;4(1):31-7.

14. Santos PHB, Alencar Filho AV, Guimarães RP, Silva CHV. Manter, reparar ou substituir restaurações? Tomada de decisão terapêutica dos estudantes do curso de Odontologia da UFPE. Int J Dent. 
2008;7(3):147-52.

15. Reis SMÁS, Oliveira AG, Lima JBG, Gonçalves LC, Gomes VL. Evolução das práticas de ensino e aprendizagem de prótese removível total na faculdade de Odontologia da UFU - um relato da experiência de seus docentes. Rev Eletrônica da Div Formação Docente. 2015;2(2):158-89.

16. Domingues GG, Fonseca GS, Zilbovicius C, Frias AC, Junqueira SR. Modalidades de ensinar e aprender: educação online no curso de graduação em Odontologia. Rev ABENO. 2016;16(1):61-72.
17. Peixoto RTRC, Gonçalves PVAJ, Alvim $\mathrm{HH}$, Amorim HCS, Araújo AVA. O emprego das tecnologias de informação e comunicação no ensino superior: relato de experiência sobre a oficina "modelo híbrido de ensino." Rev Docência Ens Sup. 2015;5(1):183-204.

Correspodência para:

Profa. Eline Cristina Gava Pizi

e-mail: elanepizi@unoeste.br

Curso de Odontologia - UNOESTE

Rua Neide Pimenta Tolomei, 46

19053-695 Presidente Prudente/SP. 